\title{
Robust Indoor Localization across Smartphone Models with Ellipsoid Features from Multiple RSSIs
}

\author{
MASATO SUGASAKI, Tokyo Institute of Technology \\ MASAMICHI SHIMOSAKA, Tokyo Institute of Technology
}

\begin{abstract}
Localization for mobile devices has become important as the basis technology for various ubiquitous computing applications. While GPS is leveraged as the de-facto standard technology in outdoor localization, its accuracy is poor indoors. For twenty years, researchers have tried to investigate indoor localization technology using fingerprinting from received signal strength indicators (RSSIs). With the widespread use of smartphones in the last decade, device dependency (e.g. antenna characteristics) must be considered to avoid performance degradation, while most of the recent localization approaches assume that all the smartphone models have the same device characteristics.

In this paper, we propose a novel feature representation based on multiple RSSIs for compensating performance degradation against smartphone models changes. In contrast to the previous feature representation based on a single RSSI, our new feature representation, which we call Ellipsoid features, employs tuples of pair of RSSIs to eliminate device dependence in the path loss model for wave propagation. In contrast to recent advances in machine learning methods such as domain adaptation, multi-task learning, and semi-supervised learning, our approach requires no additional dataset nor retraining for the new target models. This simplicity would promote ubiquity of indoor localization in the era of smartphones. Moreover, our feature representation works well compared to the state-of-the-arts in feature representations based on multiple RSSIs even when only a small number of access points (APs) are available. Experimental result using smartphone devices including Android Nexus5, Nexus5X, Nexus6P, and Xperia X Performance shows that our approach achieves superior performance over the state-of-the-art indoor localization models as well as robust performance against device changes.
\end{abstract}

CCS Concepts: •Networks $\rightarrow$ Location based services; $・$ Information systems $\rightarrow$ Location based services;

General Terms: EXPERIMENTATION

Additional Key Words and Phrases: Location fingerprint, Wi-Fi, indoor localization, positioning system, RSSI variance problem

ACM Reference format:

Masato Sugasaki and Masamichi Shimosaka. 2017. Robust indoor localization across smartphone models with ellipsoid features from multiple RSSIs. PACM Interact. Mob. Wearable Ubiquitous Technol. 1, 3, Article 103 (September 2017 ), 16 pages. DOI: http://doi.org/10.1145/3130968

\section{INTRODUCTION}

Recently, localization for mobile devices has become important as the basis technology for various ubiquitous computing and applications such as Location-Based Services (LBS), and navigation. Currently, localization by GPS is the main stream as outdoor localization [10] while GPS provides less accuracy in indoor situation. From the pioneering work by Bahl (RADAR [1]), indoor localization using Received Signal Strength Indication (RSSI) has been explored as one of the central issues in ubiquitous computing community.

Permission to make digital or hard copies of all or part of this work for personal or classroom use is granted without fee provided that copies are not made or distributed for profit or commercial advantage and that copies bear this notice and the full citation on the first page. Copyrights for components of this work owned by others than the author(s) must be honored. Abstracting with credit is permitted. To copy otherwise, or republish, to post on servers or to redistribute to lists, requires prior specific permission and/or a fee. Request permissions from permissions@acm.org.

(C) 2017 Copyright held by the owner/author(s). Publication rights licensed to ACM.

2474-9567/2017/9-ART103 \$15.00

DOI: http://doi.org/10.1145/3130968

Proceedings of the ACM on Interactive, Mobile, Wearable and Ubiquitous Technologies, Vol. 1, No. 3, Article 103. Publication date: September 2017. 
In the literature, tuples or vectors, where each score represents an RSSI from a single access point (AP), are often leveraged as inputs of indoor localization [1][19][11][21]. This simple representation promotes the localization performance thanks to the advances in machine learning techniques; however, the performance is not always good over the devices changes. This stems from the fact that the distribution of a single RSSI varies across the device types (e.g. the antenna characteristic of Android Nexus5X differs from that of Nexus6P). On the other hand, in many research on indoor localization based on fingerprints, it is assumed that the RSSIs acquired by all the devices follow the same distribution [16]. This is equivalent to the assumption that the radio reception characteristics of the device used in the training the model and the devices used in the market are equal. In the era of smartphones with various models on the market, the above assumption on the distribution is not satisfied.

In order to deal with RSSI distribution changes across the smartphone models, completely new calibration process (i.e. acquiring RSSI signals annotated with location labels) for new the target device on the market can be employed as a naive approach. However, this is a time-consuming and labor-intensive process. Such large calibration costs hinder the spread of indoor localization technology. For this issue, transfer learning [3] or domain adaptation [20] can be adapted as recalibrating the localization model with a small number of training data on the new target devices. These machine learning technologies are expected to reduce the labor tasks; however, we still have to provide additional fingerprinting data with supervision about location. Additionally, this cannot provide any guarantees of removing the performance degradation across the devices. Therefore, an approach without any additional data acquisition or retraining process for the device dependency issue should be investigated.

Recently, robust indoor localization across the device types is explored by a designing feature representations with discriminative machine learning approaches [18][22][23]. Thanks to its optimization process, this framework helps us to design the effective features by observing the localization performance. As a state-of-the-art feature representation, that is insensitive across the smartphone models, Zheng et al. [23] proposed a new binary feature representation constructed from the magnitude comparison between two RSSIs. Thanks to the property of the path-loss model of wave propagation, each element in this feature representation is insensitive to the devices. This feature representation accelerates the robustness over device changes. However, this model is not robust under the environment where only a small number of APs are available.

In this paper, we propose a new feature representation from multiple RSSIs for pursuing the robustness of indoor localization against smartphone models changes even if only small number of APs are available. Our contributions of this paper are summarized as follows:

(1) Our work provides first comprehensive analysis of the radio propagation model for designing a new feature representation robust against smartphone models changes.

(2) We propose a novel feature representation, named as the Ellipsoid feature, that is robust against device changes even if the small number of APs are available. We also give qualitative analysis on the superiority of our new model over the state-of-the-arts with synthetic simulation. The simulation result implies that our model requires less number of APs than state-of-the-arts for competitive performance.

(3) Experimental result using a real world mobile-phone RSSI dataset including four device categories shows that our novel feature representation achieves the best performance over the state-of-the-art as well as robust against device changes.

\section{Related work}

In the last decade, many researchers have investigated to resolve the performance degradation issue caused by the various changes from the calibration (e.g. the environmental changes due to the furniture moves) [2,13-15], where transfer learning, domain adaptation are often leveraged as key technology.

Due to the fact that the RSSI distribution changes caused by environmental changes through time-shift, the advanced machine learning techniques were proven to be to reduce the labor tasks for re-calibration process.

Proceedings of the ACM on Interactive, Mobile, Wearable and Ubiquitous Technologies, Vol. 1, No. 3, Article 103. Publication date: September 2017. 


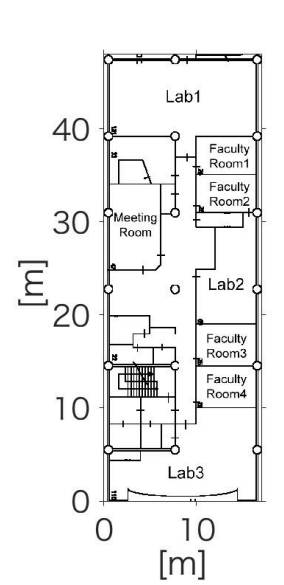

(a)

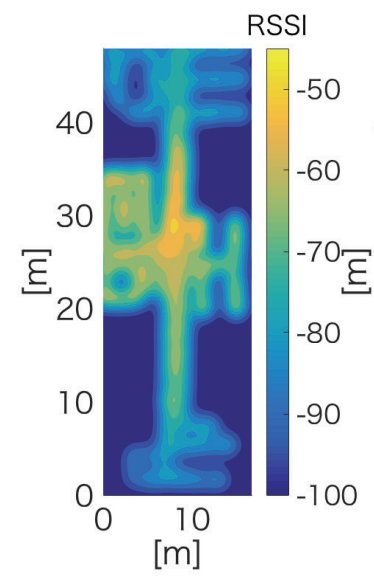

(b)

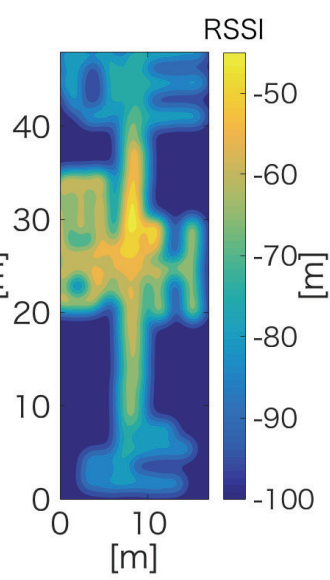

(c)



(d)

Fig. 1. RSSI distribution on different devices for 1 AP (a)Map (b)The RSSI distribution of Nexus5 (c)The RSSI distribution of Nexus9 (d)RSSI difference between acquired by Nexus5 and Nexus9

Similar to performance degradation over time changes, we have to focus on robustness against device changes in the era of smartphones (e.g. iPhones, Android). This stems from the fact that the antennas of these devices are different from each other. Therefore, it is important to consider the device dependency of the localization models as well as the time change of the environment [15][14][13]. Fig. 1 visualizes the RSSI distribution of two target models by using the Gaussian processes [9]. This shows that the difference between the RSSIs is $10 \mathrm{dBm}$ at the maximum. This difference of RSSI between the models causes a large error in localization.

Similar to using transfer learning across the time shift, a method using transfer learning efficient recalibration method across the devices is proposed recently [22][24]. These researches can make a new localization model for each device by using a small amount of target device data. However, at the present time when various smart devices are commercially available, it is not feasible to acquire the data for each device, respectively. Therefore, it is not feasible to apply transfer learning to the device dependency issue due to the nature of the problem. Thus, it is necessary to eliminate device dependency from the other perspective.

As an alternative to advanced machine learning techniques, recent work focuses on constructing novel feature representations in order to remove the device dependency issue [18][23]. Kjaergaard et al. [18] and Zheng et al. [23], which are closest to our work, investigate to resolve device dependency issue by using a low device dependency feature representation. Kjaergaard et al. [18] tried to solve the device dependency by adopting the ratio of the two RSSIs from two APs as one element of the feature vector. However, the device dependency was not completely canceled due to the maximum score used in the feature representation was caused by device dependent characteristics.

Zheng et al. [23] also tried to resolve the device dependency with a feature vector constructed from binary scores where the score is obtained from a comparison of RSSIs from two APs. They used distance relationships as a feature quantities to eliminate device dependency. However, their binary representation drops off the indication how close AP is. Thus, this feature representation does not provide robustness under the situation where the number of available APs is small. To deal with this issue, Zheng et al. [23] also provides alternative feature representation obtained from three or more APs. However, it is not feasible in computational cost since it causes the exponential growth in the dimensions of this feature. 
Dong et al. [4] and Fang et al. [8] used alternative approaches for feature representation to resolve the device dependency issue. However, they also got stuck in device dependency in terms of wave propagation equation.

Our model, which is akin to Dong et al. [4] in terms of using differentiation of RSSIs, achieves as good cancellation as Zheng's model, while the necessary number of APs are less required than Zheng's model. As we mentioned in the following sections, the same feature score obtained from the two APs in our representation can be projected onto ellipsoid boundaries in 2D localization space, whereas the binary representation proposed by Zheng represents a line. Thus, we term this novel feature representation the ellipsoid feature.

The rest of this paper is organized as follows. In Section 2, formalization of indoor localization by multi-class classifier on discriminative model and the existing feature representation using RSSI from device dependency perspective are given. Section 3 presents our novel feature representation derived from a path loss model for wave propagation to resolve device dependency. In Section 4, we describe the experiments for verifying our model. Finally, Section 5 presents the conclusion of this study.

\section{FEATURE REPRESENTATION FOR INDOOR LOCALIZATION BASED ON RSSI}

In this section, we briefly describe a formulation of RSSI based localization. Then we provide basic feature representations and their problems in terms of the device dependency.

\subsection{Formulation of indoor localization}

As a basis method of localization, the two approaches are categorized as follows. One is a generative model that predicts RSSI from the probabilistic properties of RSSI by such as Gaussian processes [9]. The other one is a discriminative model that constructs a model directly from the acquired supervised data with a feature representation. Moreover, the discriminative approach has two frameworks in the discriminative approach. The first framework can be casted into regression form wherein the system infers 2D coordinates from the RSSI signals [24]. The second one is a classification basis for estimating the posterior probability of each position label [16]. In this research, we consider a type of classification problem in a discriminative framework that makes it simple to discuss the feature representation. Note that our result is not limited to the case of classification but can be applied to the regression case.

In this paper, let $d \in \mathbb{N}$ be the number of available APs in the environment. Each smartphone obtains a RSSI fingerprint score as $\boldsymbol{x}^{\top}=\left(x_{1}, \ldots, x_{d}\right)$, where $x_{i}$ is the RSSI acquired from the $i$-th AP. If the $i$-th AP is not observed, we define $x_{i}=\mathrm{V}$ to be a constant value $\mathrm{V} \in \mathbb{R}$. We consider the classifier $f$ as the following mapping function:

$$
f(\boldsymbol{x}): \mathbb{R}^{d} \rightarrow \mathcal{L}
$$

where $\mathcal{L}=\left\{l ; g(y)=l, g: \mathbb{R}^{2} \rightarrow \mathcal{L}\right\}$ is a collection of discrete labels about locations and $g(y)$ is a quantization function mapping from coordination space $\mathbb{R}^{2}$ to label space $\mathcal{L}$. Therefore, we construct $f$ as

$$
\begin{aligned}
& f(\boldsymbol{x})=\underset{l}{\arg \max } f_{l}(\boldsymbol{x}), \\
& f_{l}(\boldsymbol{x})=\boldsymbol{\theta}_{l}^{\top} \boldsymbol{\phi}(\boldsymbol{x}),
\end{aligned}
$$

where $\phi$ is the feature vector constructed from RSSIs and $\theta_{l}$ is the parameters for location $l$.

Prior to describing the existing techniques of RSSI feature representation, let us describe the reason why we adopt the discriminative framework in our paper. This stems from the fact that the optimization can be executed through any feature representations and this helps us to design a better feature representation. Specifically, once the classifier gets higher performance through the dataset $\mathcal{D}=\left\{\boldsymbol{x}^{(k)}, y^{(k)}\right\}_{k=1}^{n}$, the features providing better results will be considered a better representation for localization. In our paper, we borrow the following basic 
optimization problem proposed in [16] for simplicity as

$$
\underset{\boldsymbol{\theta}_{1, \ldots,|\mathcal{L}|}}{\arg \min } \frac{1}{N} \sum_{k=1}^{|\mathcal{D}|} \Delta\left(g\left(y^{(k)}\right), f\left(\boldsymbol{x}^{(k)}\right)\right)+\sum_{l \in \mathcal{L}} R\left(\boldsymbol{\theta}_{l}\right),
$$

where $\Delta$ is a cost function defined as the distance and $R\left(\theta_{l}\right)$ is regularization term. In this paper, we employ a cost function based on L2 distance and a L2 norm for regularization term similar to the setting in [24]. Note that this problem can be effectively optimized by using recent advances in stochastic gradient methods such as FOBOS [6], AdaGrad [5], and Adam [17]. Note that this optimization problem can be available in any feature representations.

\subsection{Device dependency based on the path loss model for radio propagation}

The device dependency of the RSSI is caused by the characteristics of the antennas and hardware of each device. RSSI $x_{i}$ of received from the $i$-th AP follows the path loss model for radio propagation [7]:

$$
x_{i}=z_{m, l_{0}}-10 \beta_{i} \log \left(\frac{l}{l_{0}}\right)+\psi,
$$

where $z_{m, l_{0}}$ is absolute gain with $l_{0}$, the referential distance on the device $m \in \mathcal{M}, l$ is the distance between the device and the AP, $\psi$ is Gaussian noise, and $\beta_{i}$ is the path loss exponent. The term $z_{m, l_{0}}$ in (5) contains the device dependencies owing to the hardware characteristics (e.g. antennas) of each device; therefore, RSSI $x_{i}$ is the device dependent in nature.

In the rest of this section, we discuss the existing feature representations and their limitation.

\subsection{Feature representation based on a single RSSI}

As a feature representation created from a single RSSI, for example, thresholding representation and Gaussian representation [16] can be a typical choice. In the thresholding model, the $j$-th feature quantity by the binary expression which threshold value is exceeded or not is expressed by following equation:

$$
\{\boldsymbol{\phi}(\boldsymbol{x})\}_{j}=\left\{\begin{array}{ll}
1 & \left(s x_{i} \geq s \tau_{j}\right) \\
0 & \left(s x_{i}<s \tau_{j}\right)
\end{array},\right.
$$

where $\tau_{j}$ is a heuristically determined threshold value and value $s$ is a sign $s \in\{ \pm 1\}$. As described in Section 2.2 , the maximum value of the RSSI depends on the device. This causes difficulty in optimizing the threshold for multiple target models without any data on the new target models. Therefore, this expression cannot be used to eliminate the device dependency.

The Gaussian representation [16] employs a Gaussian radial basis function (GRBF) to map raw values to the values which allows to have widths in near values. The $j$-th feature is expressed as

$$
\left.\{\boldsymbol{\phi}(\boldsymbol{x})\}_{j}=\exp -\frac{\left(x_{i}-\mu_{j}\right)^{2}}{2 \sigma_{j}^{2}}\right),
$$

where $\mu_{j} \in \mathbb{R}$ is mean of the GRBF and $\sigma_{j}>0 \in \mathbb{R}$ is a hyper-parameter indicating a measure for the RSSI value. Similar to the thresholding model, the GRBF approach also gets stuck from the device characteristic without the data on the new devices.

\subsection{Feature representation based on multiple RSSIs}

As a basis model of our new feature representation, this section briefly describes the recent work and its problems of feature representation using multiple RSSIs to tackle the device dependency. 




(a) Outline of the binary feature representation : Binary in figure represents the redundant of binary narrowing down.

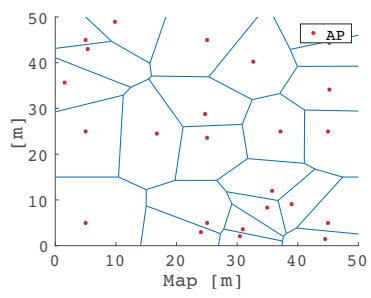

(b) Voronoi regions under 25-th APs

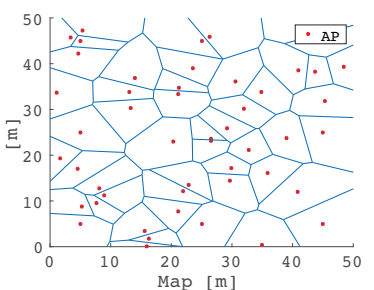

(c) Voronoi regions under 50-th APs

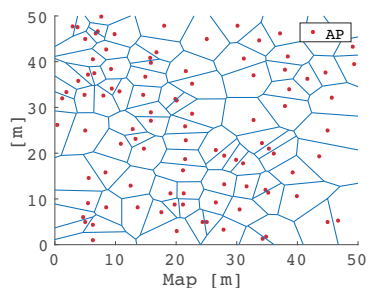

(d) Voronoi regions under 100-th APs

Fig. 2. Simulation result of feature representation with binary expression

2.4.1 Feature representation using ratio of two RSSIs. Kjaergaard et al. used the ratio of two RSSIs for feature representation to reject device dependency. This approach resolves the device dependency by the ratio of two RSSIs and normalizes that by using the maximum value of the RSSI obtained for each device. The $j$-th feature is expressed as

$$
\{\boldsymbol{\phi}(\boldsymbol{x})\}_{k}=\log \left(\frac{x_{i}}{x_{j}}\right)-\log \left(\frac{1}{\max x_{m}}\right),
$$

where $\max x_{m}$ is the maximum value of the RSSI received by device $m \in \mathcal{M}$. However, the maximum score of RSSIs is not device independent but inherit from the device characteristics; the resultant feature score is also device dependent as in (5). Needless to say, the maximum score cannot be obtained precisely prior to obtaining the brand new smartphones.

2.4.2 Feature representation using relation about the multiple RSSIs. Zheng et al. used a the binary feature representation that expresses the relation based on the difference between the two RSSI values to reject the device dependency. This relation indicates that which AP is closer to a straight line bisecting the environment as shown Fig. 2(a). In this feature descriptor, the $k$-th score in the feature is constructed from the $i$-th and $j$-th APs in the feature is expressed by the following equation as

$$
\{\boldsymbol{\phi}(\boldsymbol{x})\}_{k}=h\left(x_{i}>x_{j}\right)
$$

where $h$ is a map function boolean to binary. As this expression divides the area into two regions from one feature score, ambiguity becomes large in principle as shown in Fig. 2(a). For this reason, a large number of straight lines are required to narrow down the position. In other words, a large number of APs are required to performing accurate localization.

Fig. 2(b) - (d) shows the simulation results of narrowing down the position by straight lines dividing up the area of positioning into two when the AP is randomly installed in $50 \mathrm{~m} \times 50 \mathrm{~m}$. We simulated this by a Voronoi region evaluated in the environment of $25 \mathrm{APs}, 50 \mathrm{APs}$ and $100 \mathrm{APs}$, which is a range close to the most target AP for each AP. As shown in Table 1, in order to suppress the error distance to about $5 \mathrm{~m}$, it needs about 100 APs.

Therefore, the feature representation by bisectors is a poor in narrowing down the position. 
Table 1. Averages and maximum value of Voronoi area area to AP number

\begin{tabular}{c|cc}
\hline \hline The Number of APs & Averages & Maximum value \\
\hline 25 & $100 \mathrm{~m}^{2}$ & $190.5 \mathrm{~m}^{2}$ \\
50 & $50 \mathrm{~m}^{2}$ & $99.5 \mathrm{~m}^{2}$ \\
100 & $25 \mathrm{~m}^{2}$ & $64.9 \mathrm{~m}^{2}$ \\
\hline
\end{tabular}

To resolve this issue, Zheng et al. also used an alternative descriptor (high order pairwise (HOP)) as

$$
\{\boldsymbol{\phi}(\boldsymbol{x})\}_{k}=h\left(\sum_{(i, j)} c_{i, j}\left(x_{i}-x_{j}\right)+b>0\right),
$$

where $c_{i, j} \in \mathbb{R}$ can be seen as the normalized number of times that $\left(x_{i}-x_{j}\right)$ is used to construct the linear combination, and $b$ is a hyperparameter for the relaxation the thresholding condition. This feature contains tuples of two pairs of APs rather than also a pair of APs binary representations. As a result of this representation, the number of features increases with the order of the exponent with respect to the number of pairs considered by the dimension. To deal with feature increasing, the feature is reduced by compressing by the nonlinear transformation such as the Boltzmann machine [12]. However, this representation causes another problem in terms of computational cost due to the nonlinear transformation.

\section{ELLIPSOID FEATURE : FEATURE REPRESENTATION WITH THE LOW DEVICE DEPENDENCY}

\subsection{Definition of Ellipsoid Feature}

This section describes our novel feature representation for RSSI localization robust to device changes. To design the new efficient feature representation that is insensitive across the devices, we focus on the subtraction value with the two APs inspired by the success of the recent progressive work. The difference in signal strength is expressed from the path loss model (5) by the following equation:

$$
\begin{aligned}
\delta x_{i, j} & =x_{i}-x_{j} \\
& =-10 \beta_{i} \log \left(\frac{l_{i}}{l_{0}}\right)+10 \beta_{j} \log \left(\frac{l_{j}}{l_{0}}\right)+\psi_{i}-\psi_{j},
\end{aligned}
$$

where $\beta_{i}$ and $\beta_{j}$ is the path loss exponent for each APs. If we assume that $\beta_{i}$ and $\beta_{j}$ is the same value $\eta_{i, j}$, (11) should be the following equation:

$$
\delta x_{i, j}=x_{i}-x_{j}=-10 \eta_{i, j} \log \left(\frac{l_{i}}{l_{j}}\right)+\psi_{i}-\psi_{j},
$$

where $\psi_{i}$ and $\psi_{j}$ are the Gaussian noise with 0 mean due to the environmental changes and other effects such as from human body. In both (11) and (12), it can be seen that the device dependent terms in (5) are canceled by subtraction. That is, the RSSI difference is expressed with less device dependent. Fig. 3 depicts the contour plot of this subtraction value used in our novel feature description and illustrates multiple bipolar lines as shown in Fig. 3. By using this value, even in a small number of APs, it is clear that the subtraction score is effective to narrow down the possible regions compared to the binary scores in Zheng's model [23].

Though the subtraction score provides effective cues for localization, the score itself is not correlated to the function value of the localization models in (11). So we employ a nonlinear feature transformation with a Gaussian 


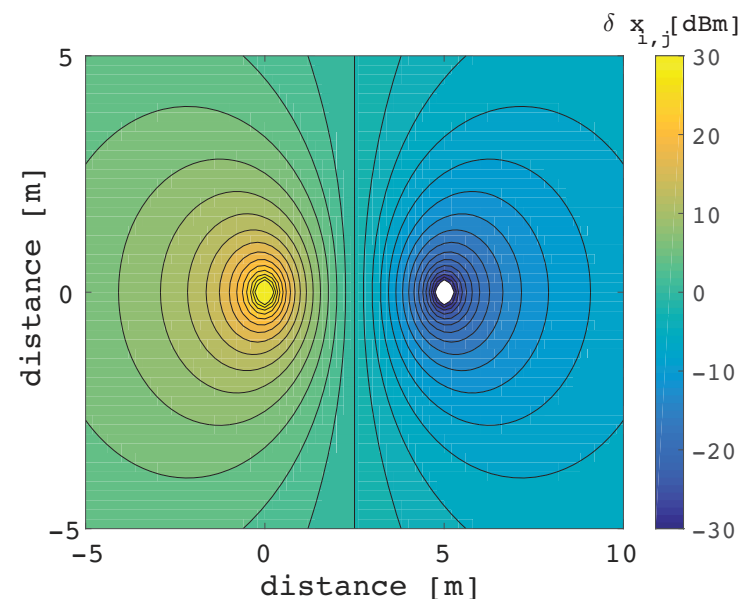

Fig. 3. Contour map of RSSI difference representation

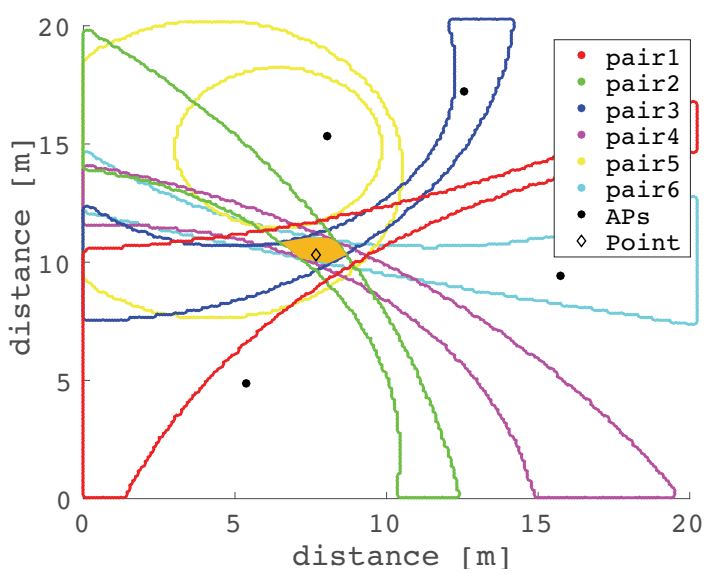

Fig. 4. Projected Ellipsoid feature with vector quantization (like Voronoi diagram) using 4 APs (6 AP pairs).

kernel inspired by nonparametric modeling. The resultant feature defined by the following equation

$$
\left.\{\boldsymbol{\phi}(\boldsymbol{x})\}_{k}=\exp -\frac{\left(\delta x_{i, j}-\mu_{k}\right)^{2}}{2 \sigma_{k}^{2}}\right),
$$

is named with ellipsoid feature, where $\mu_{k} \in \mathbb{R}$ is mean of GRBF, $\sigma_{k}>0$ is a hyper-parameter indicating a measure of the RSSI value. By using this transformation, the tuples of this feature representation selects the region covered by multiple ellipsoid lines as in Fig. 4. In each region closed by ellipsoid lines, the feature scores have similar to the others whereas the different feature values can be casted into other regions.

Though we focus on the relative distance from the two APs, our model is distinct from the traditional triangular models. Since the triangular models convert distances from single RSSIs, it gets stuck from device dependency in nature. In contrast to the triangular models, our model is quite free from the device dependency.

\subsection{Simulation result on Ellipsoid feature}

To verify the efficiency of our model, we conducted a simulation to estimate how well the possible location candidates can be narrowed down through the encoded scores of ellipsoid feature. In this simulation, we assume four APs were installed in the environment. When we focus on the distributions of the feature score of all the six pairs in (11), the possible locations similar to the feature score obtained from the point is limited to the combination of the ellipsoid lines (the orange colored region) as shown in Fig. 4.

To verify the efficiency of our model quantitatively, we compare the areas of Voronoi-like regions depicted from the feature scores. In this simulation study, we set the sigma in the ellipsoid feature $\sigma 5$ and mean of the ellipsoid feature $\mu$ from 30 to -30 by 5 . The simulation results shows that the maximum area can be narrowed down to approximately $31 \mathrm{~m}^{2}$ even if the number of APs is limited to four. Compared with the binary representation that using the distance relation about two APs in Fig. 2(c), the maximum area narrowed down is $64.9 \mathrm{~m}^{2}$ at $100 \mathrm{AP}$, our proposed feature representation can narrow down the position with a small number of APs. This simulation study implies that our model can achieve efficient localization with accuracy even if the number of APs is quite limited. 


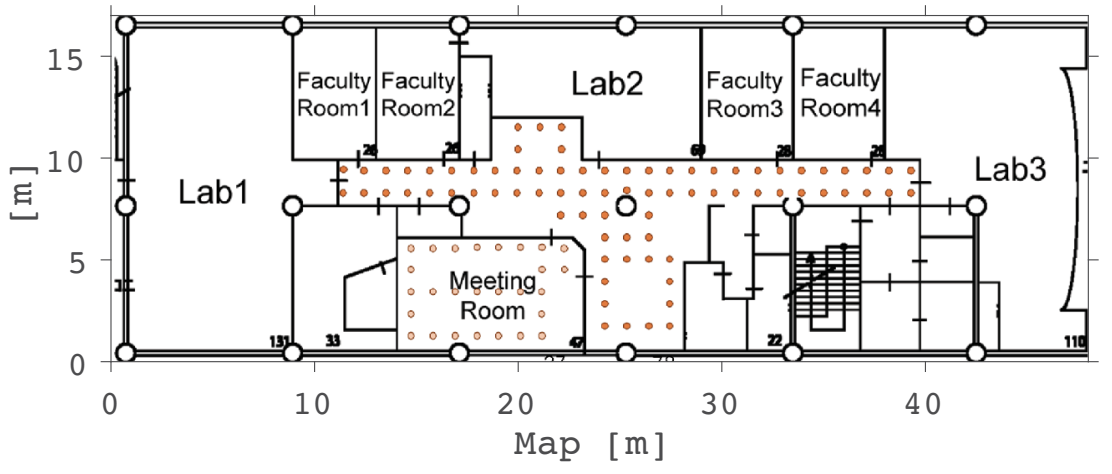

Fig. 5. Map of the environment for experiment

\section{EXPERIMENT}

In this section, we verify the performance our new feature representation compared with the state-of-the-arts for device dependency issues.

\subsection{Experiment Setting}

4.1.1 Dataset. To verify the performance, we obtain the RSSI signals across devices over the $15 \mathrm{~m} \times 40 \mathrm{~m}$ on one floor of a building located on a university campus. We set to the target area for this experiment to one conference room that size is $6 \mathrm{~m} \times 9 \mathrm{~m}$ and corridors that length is $27 \mathrm{~m}$. The target area of this experiment is $10 \mathrm{~m} \times 27 \mathrm{~m}$ in total. To obtain the data, we set 105 points on a grid which is $1 \mathrm{~m} \times 1 \mathrm{~m}$ on the floor without furniture or obstacles. We leverage Nexus5, Nexus9, Nexus5X, and Xperia X Performance as the devices for this dataset. We acquire the data 10 times at each point per device per day. This is iterated through 5 days for canceling the dataset biases. The dataset contains 5250 pairs of RSSI fingerprint vectors and location labels in total. For each device of the dataset, five data at each point are used for learning, five data of each point are used for verification, and data of 40 points at each other point are used for testing.

4.1.2 Comparison methods. For the comparison, we prepare four feature representations as the compared models. The first one is using RSSI directly (UniNaive), the second one is using single RSSI expressed by GRBF (UniGaussian) [16], the third one is directory using differential value of RSSI directory (PairNaive) [4], and the fourth one is using a binary that indicates which AP of the pair is close to the AP (PairBinary) [23].

For the setting of the UniGaussian feature, we used seven GRBFs with all pair about mean of from -35 to -95 by 10 and variance of $\sigma=1.0$ in the hyperparameter. For the setting of the proposed Ellipsoid feature, we used fifteen GRBFs with all pairs of means from -70 to 70 by 10 and variance of $\sigma=1.0$ in the hyperparameter.

4.1.3 Evaluation metric. We evaluated the result by the error function $e(y, \hat{y})$ by true position $y$ and estimated position $\hat{y}$ as

$$
e(y, \hat{y})=\sqrt{\left(l_{x}-\hat{l}_{x}\right)^{2}+\left(l_{y}-\hat{l}_{y}\right)^{2}}
$$

To verify the result, we employ the value of the average error distance, 25-th percentile error distance, median, and 75-th percentile error distance about the results. We also evaluated the CDF curves for visualizing the localization performance. 


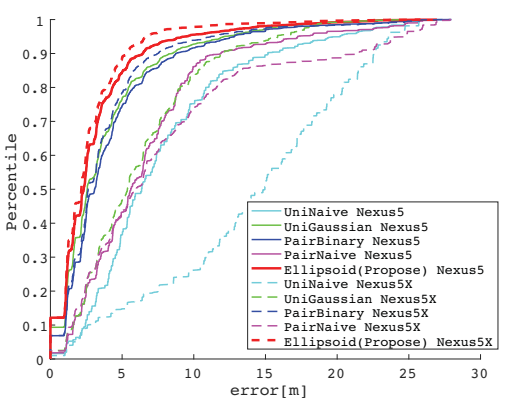

(a)

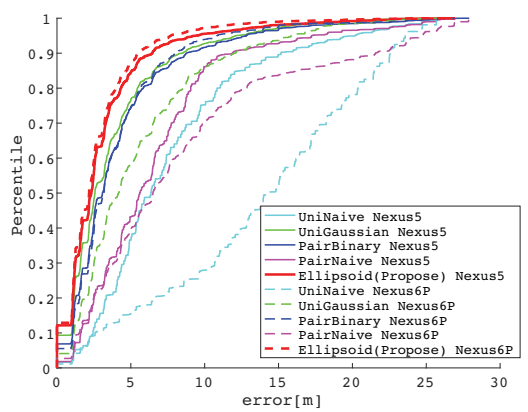

(b)

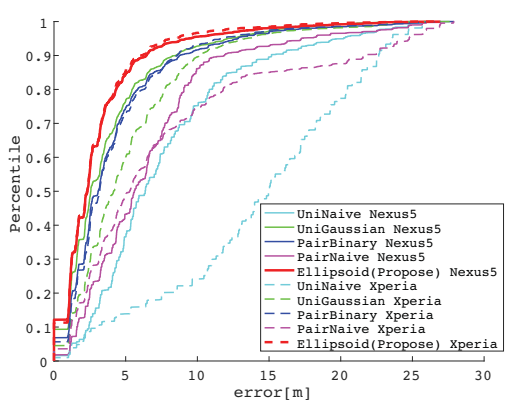

(c)

Fig. 6. CDF trained with Nexus5 (a)Target : Nexus5X (b)Target : Nexus6P (c)Target : Xperia

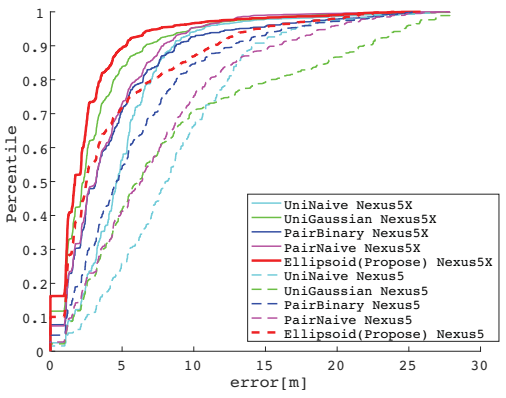

(a)

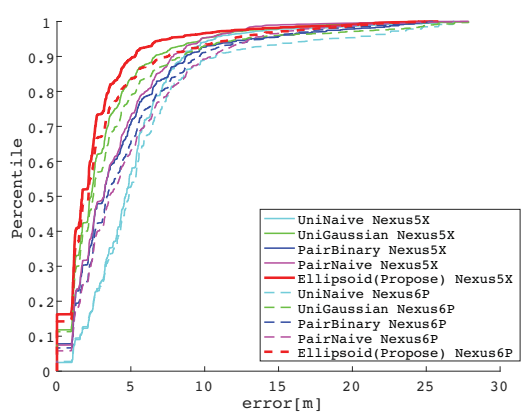

(b)

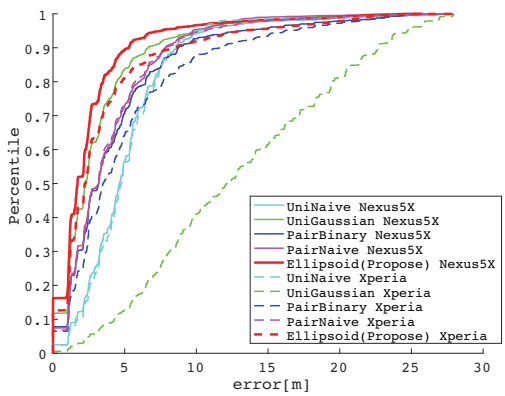

(c)

Fig. 7. CDF trained with Nexus5X (a)Target : Nexus5 (b)Target : Nexus6P (c)Target : Xperia



(a)

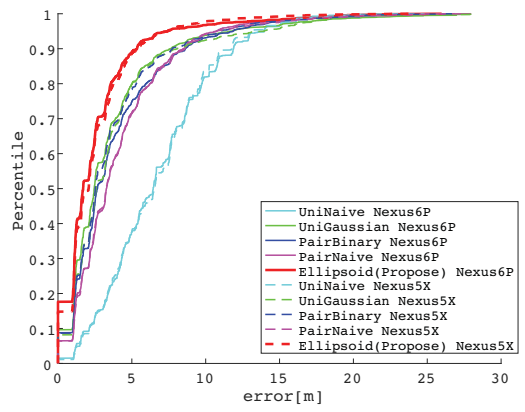

(b)

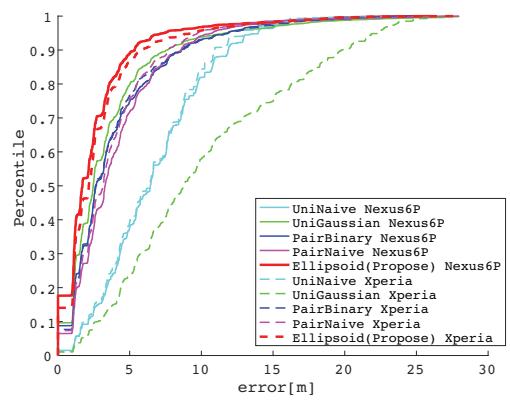

(c)

Fig. 8. CDF trained with Nexus6P (a)Target : Nexus5 (b)Target : Nexus5X (c)Target : Xperia

\subsection{Results}

From Fig. 6 to 9, the red solid and dotted lines show the results of the proposed method. Moreover, the solid line represents the localization result of the device used for learning, and the dotted line indicates the localization 


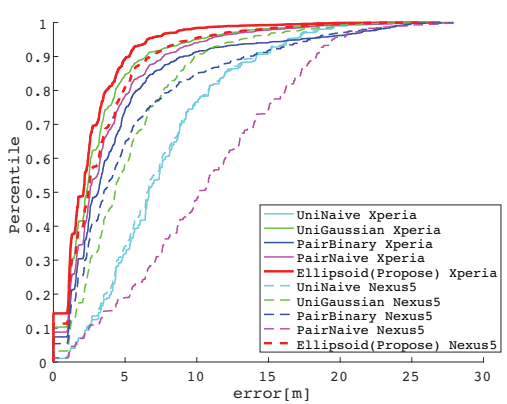

(a)

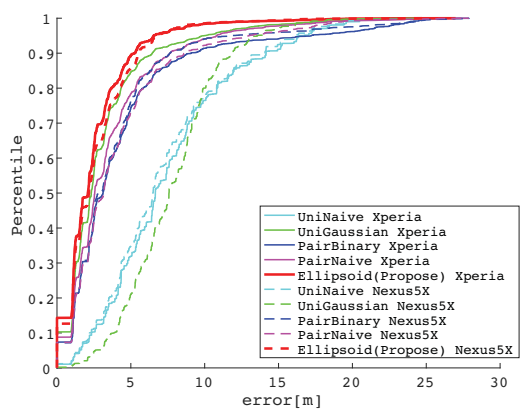

(b)

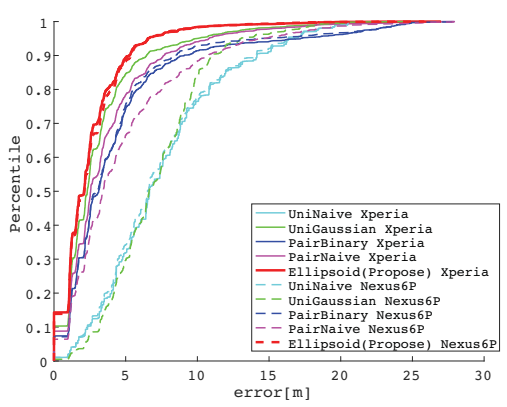

(c)

Fig. 9. CDF trained with Xperia (a)Target : Nexus5 (b)Target : Nexus5X (c)Target : Nexus6P

result of the terminal not used for learning. In these figures, if the CDF curve is closer to the upper left side, it implies that the method achieves better performance. From Fig. 10 to 13, they show the boxplots in the respective localization results.

From Fig. 6 to 9, the red solid line and the dotted line representing the proposed method are all closest to the upper left for all devices. From this fact, it was shown that the proposed method can perform localization with the highest accuracy in the situation that we can obtain the smartphone devices a priori and use it in the training process.

When observing quantitatively, focusing on the localization result for training with Nexus5 data in Fig. 10 as a representative, the average error of the proposed method is $3.11 \mathrm{~m}$, PairBinary method is $4.20 \mathrm{~m}$, PairNaive method is $6.60 \mathrm{~m}$, UniGaussian method is $3.82 \mathrm{~m}$, and UniGaussian method is $7.70 \mathrm{~m}$. Thus, our proposed method outperforms the other methods on average. Moreover, focusing on the 75 th percentile value, the result shows that the proposed method is also lower than the other methods. From these results, the proposed method performs accurate localization even in the existing framework without model transfer.

From the view point of the device dependency issue, as shown in Fig. 6 to 9, the CDF curve without model transfer (solid line) and the CDF curve with model transfer (dotted line) is overlapped. Quantitatively, focusing on the performance of Nexus6 6 trained with the data obtained with Nexus5 in Fig. 10, the average error of the proposed method is $2.77 \mathrm{~m}$, while PairBinary method is $4.07 \mathrm{~m}$, PairNaive method is $8.52 \mathrm{~m}$, UniGaussian method is $5.50 \mathrm{~m}$, and UniGaussian method is $13.74 \mathrm{~m}$. Thus, even when indoor localization is performed on a device that is not gathered the training data, the error average is much smaller compared to the existing methods. The increase of error with the proposed method is $-0.34 \mathrm{~m}$, while PairBinary method is $0.13 \mathrm{~m}$, PairNaive method is $1.92 \mathrm{~m}$, UniGaussian method is $1.68 \mathrm{~m}$, and UniGaussian method is $6.04 \mathrm{~m}$. Qualitatively, the performance with feature obtained from multiple RSSIs in the case of model transfer is better than feature obtained from single RSSIs. Therefore, the proposed model achieves robust against smartphone models changes as well as achieving the better performance without model transfer.

In the method (UniNaive, Pair Naive) which uses the acquired RSSIs directly as the feature score, it can be confirmed from the result of low accuracy. This implies that the RSSI itself is less correlated to the posterior distribution of the linear classifier. From this result, the performance of the feature values obtained from a linear transformation such as work by [4] get the poorer performance than ours. Thus, we need nonlinear transformation to improve the accuracy.

Here, when we use Nexus5 as the target device while Nexus5X is used for training the accuracy gets lower compared to the accuracy of the training device. This might be caused by the difference in the number of APs 


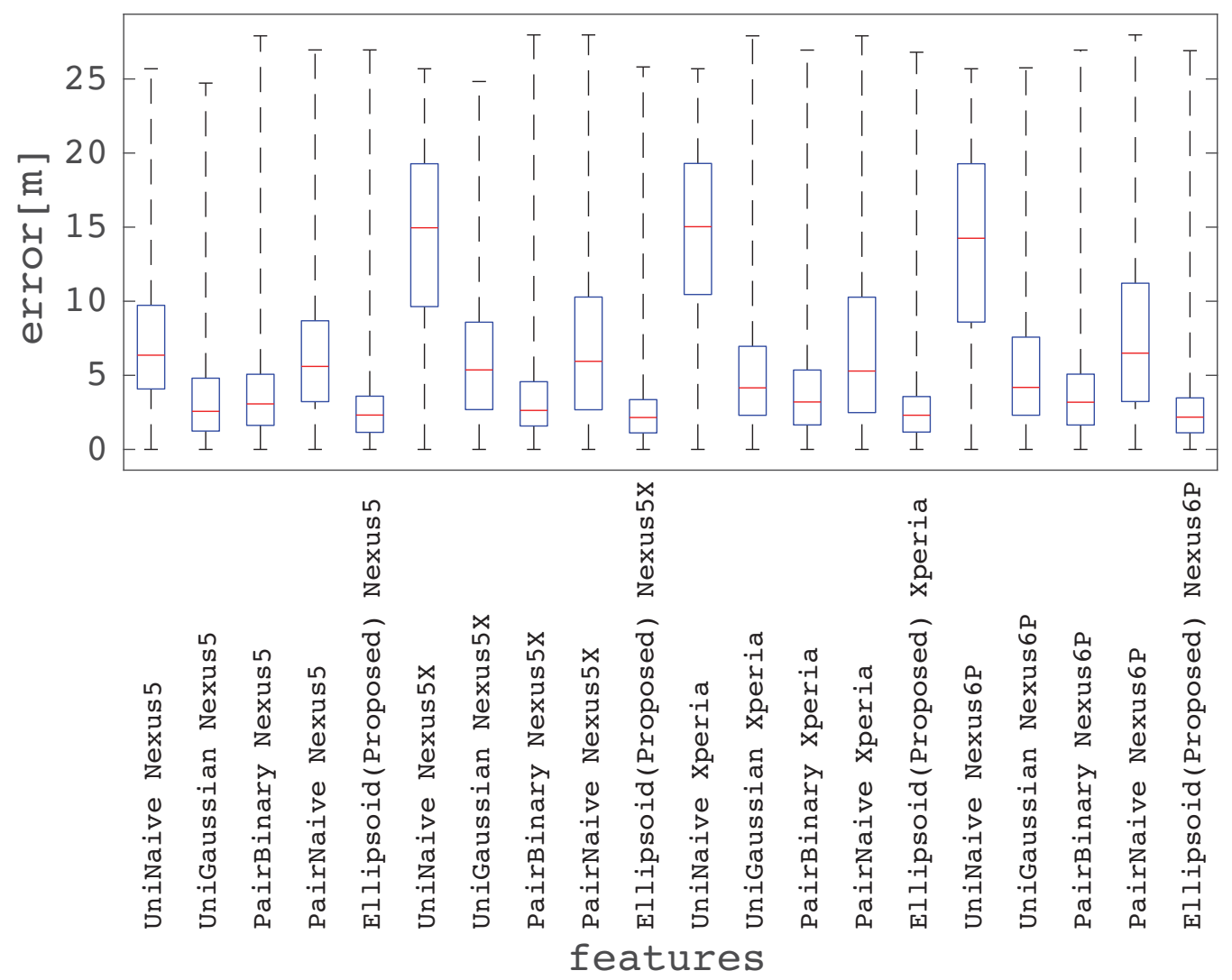

Fig. 10. Boxplots of localization errors trained with Nexus5

that can be found due to the difference in the antenna sensitivity of the devices. To resolve this issue, we insert a constant value for complementing RSSI on undiscovered APs, however it might cause performance degradation. We need further investigation to resolve this issue. That is, meaning that we need to investigate the device dependency issue including the number of observed APs as well as the distributions of RSSI in the observed APs.

We should also pay attention to the number of feature dimensions increasing. The number of the feature dimensions is in $O\left(d^{2}\right)$ due to the use of tuples of the pairs of APs. Overfitting issues due to the large number of feature dimension must be taken care of compared to the single RSSI based feature representations.

\section{CONCLUSION}

In this paper, we address the issue of performance degradation in RSSI fingerprint localization across the models in the smartphone era. To solve this problem, we provide a comprehensive review for device dependency in terms of wave propagation and derive a new powerful yet simple feature representation, what we call ellipsoid feature, which is robust across the smartphone models. In contrast to leveraging advanced machine learning techniques such as transfer learning, and domain adaptation, our model does not require any additional data collection or 


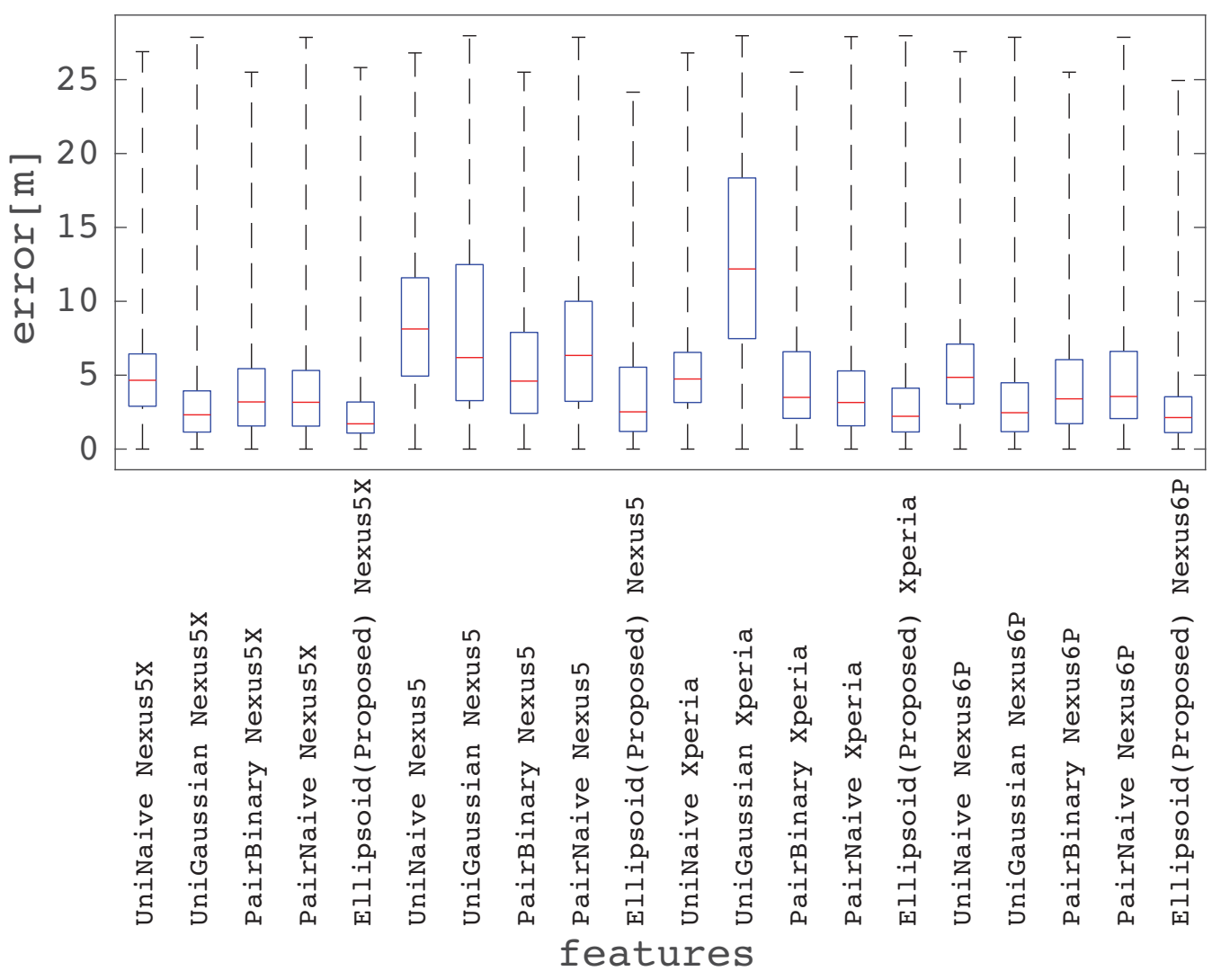

Fig. 11. Boxplots of localization errors trained with Nexus5X

retraining process for the new target devices. Compared to the state-of-the-art feature representations for the device dependency issue proposed recently, our model works well even if the number of available APs are limited. We confirmed that the proposed method is highly accurate compared to the other models for data obtained from the four types of devices. In future work, it is necessary to construct a method to solve the device dependency on the number of discovered APs. For simplicity, we employ RBF function to employ nonlinear property between the location and RSSIs, the performance with the subtracts of RSSIs from the multiple APs can be improved via recent advances in deep learning architectures, so this might open a new problem of indoor localization.

\section{ACKNOWLEDGEMENT}

This work was partly supported by CREST, JST and by JSPS KAKENHI Grant Number JP25700026.

\section{REFERENCES}

[1] P. Bahl and V. N. Padmanabhan. RADAR: An in-building RF-based user location and tracking system. In Proc. of INFOCOM2000, volume 2, pages $775-784$.

Proceedings of the ACM on Interactive, Mobile, Wearable and Ubiquitous Technologies, Vol. 1, No. 3, Article 103. Publication date: September 2017. 


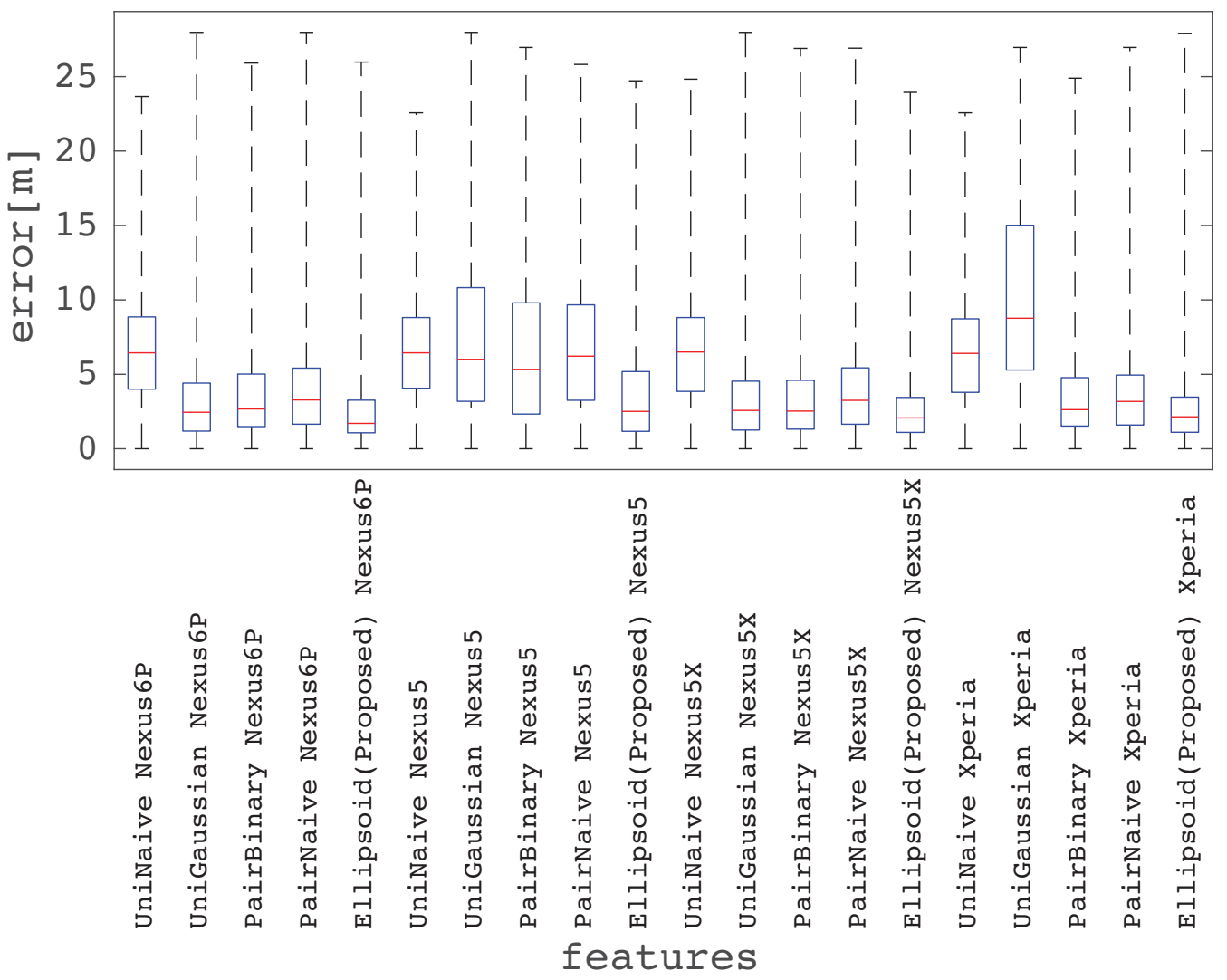

Fig. 12. Boxplots of localization errors trained with Nexus6P

[2] Y.-C. Chen et al. Sensor-assisted Wi-Fi indoor location system for adapting to environmental dynamics. In Proc. of MSWiM2005, pages $118-125$.

[3] H. Daumé III. Frustratingly easy domain adaptation. arXiv preprint arXiv:0907.1815, 2009.

[4] F. Dong et al. A calibration-free localization solution for handling signal strength variance. In Mobile Entity Localization and Tracking in GPS-less Environnments 2009, pages 79-90.

[5] J. Duchi et al. Adaptive subgradient methods for online learning and stochastic optimization. fournal of Machine Learning Research, 12(Jul):2121-2159, 2011.

[6] J. Duchi and Y. Singer. Efficient online and batch learning using forward backward splitting. fournal of Machine Learning Research, 10:2899-2934, 2009.

[7] V. Erceg et al. An empirically based path loss model for wireless channels in suburban environments. IEEE fournal on selected areas in communications, 17(7):1205-1211, 1999.

[8] S.-H. Fang and C.-H. Wang. A novel fused positioning feature for handling heterogeneous hardware problem. IEEE Trans. on Communications 2015, 63(7):2713-2723.

[9] B. Ferris et al. Wifi-slam using gaussian process latent variable models. In Proc. of IFCAI2007, pages 2480-2485.

[10] D. Han et al. Building a practical wi-Fi-based indoor navigation system. IEEE Pervasive Computing, 13(2):72-79, 2014.

[11] S. He and S.-H. G. Chan. Wi-Fi fingerprint-based indoor positioning: Recent advances and comparisons. IEEE Communications Surveys \& Tutorials 2016, 18(1):466-490

Proceedings of the ACM on Interactive, Mobile, Wearable and Ubiquitous Technologies, Vol. 1, No. 3, Article 103. Publication date: September 2017. 
Robust Indoor Localization across Smartphone Models with Ellipsoid Features from Multiple RSSIs • 103:15

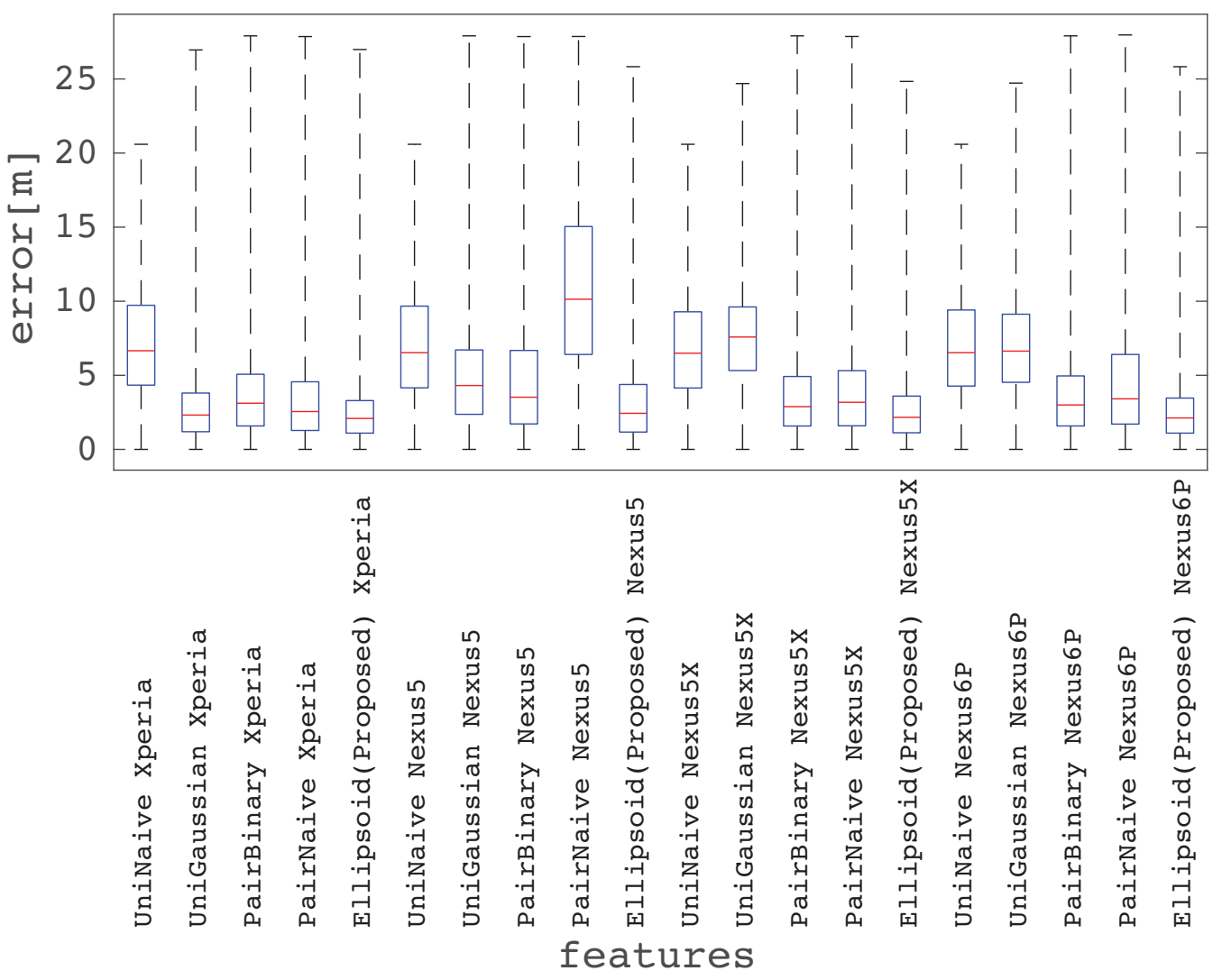

Fig. 13. Boxplots of localization errors trained with Xperia

[12] G. Hinton. A practical guide to training restricted boltzmann machines. Momentum, 9(1):926, 2010.

[13] M. K. Hoang et al. Aligning training models with smartphone properties in Wi-Fi fingerprinting based indoor localization. In Proc. of ICASSP2015, 2015.

[14] A. M. Hossain et al. SSD: A robust RF location fingerprint addressing mobile devices' heterogeneity. IEEE Trans. on Mobile Computing 2013, 12(1):65-77.

[15] H. Kashima et al. A semi-supervised approach to indoor localization estimation. In IEEE ICDM Data Mining Contest 2007.

[16] R. Kawajiri et al. Frustratingly simplified deployment in WLAN localization by learning from route annotation. In Proc. of ACML2012, pages 191-204.

[17] D. Kingma and J. Ba. Adam: A method for stochastic optimization. arXiv preprint arXiv:1412.6980, 2014.

[18] M. B. Kjærgaard and C. V. Munk. Hyperbolic location fingerprinting: A calibration-free solution for handling differences in signal strength (concise contribution). In Proc. of PerCom2008, pages 110-116.

[19] H. Liu et al. Survey of wireless indoor positioning techniques and systems. IEEE Trans. on SMC(C) 2007, 37(6):1067-1080.

[20] M. Sugiyama et al. Direct importance estimation with model selection and its application to covariate shift adaptation. In Proc. of NIPS2008, pages 1433-1440.

[21] J. Xiao et al. A survey on wireless indoor localization from the device perspective. ACM Computing Surveys (CSUR) 2016, 49(2):25.

[22] K. Zhang et al. Covariate shift in Hilbert space: A solution via sorrogate kernels. In Proc. of ICML2013, pages 388-395.

[23] V. W. Zheng et al. Cold-start heterogeneous-device wireless localization. In Proc. of AAAI2016.

Proceedings of the ACM on Interactive, Mobile, Wearable and Ubiquitous Technologies, Vol. 1, No. 3, Article 103. Publication date: September 2017. 
103:16 - M. Sugasaki and M. Shimosaka

[24] V. W. Zheng et al. Transferring multi-device localization models using latent multi-task learning. In Proc. of AAAI2008, pages 1427-1432.

Received May 2017; accepted July 2017

Proceedings of the ACM on Interactive, Mobile, Wearable and Ubiquitous Technologies, Vol. 1, No. 3, Article 103. Publication date: September 2017. 